Tables: 1

Figures: 2

\title{
The network structure of ICD-11 Adjustment Disorder: A cross-cultural comparison of three African countries
}

Yafit Levin, $\mathrm{PhD}^{1}$, Rahel Bachem, $\mathrm{PhD}^{1}$, Thanos Karatzias, $\mathrm{PhD}^{2,3}$, Mark Shevlin, $\mathrm{PhD}^{4}$, Andreas Maercker $\mathrm{PhD}^{1}$, Menachem Ben-Ezra, $\mathrm{PhD}^{5}$,

${ }^{2}$ Edinburgh Napier University, Edinburgh, Scotland, UK

${ }^{3}$ NHS Lothian, Rivers Centre for Traumatic Stress, Edinburgh, Scotland, UK

${ }^{4}$ Psychology Research Institute, School of Psychology, Ulster University, Northern Ireland

${ }^{5}$ School of Social Work, Ariel University, Ariel, Israel

${ }^{1}$ Corresponding author

Dept. of psychology, Division of psychopathology and clinical intervention

Zurich, Switzerland

ohanayaf@gmail.com

972-547957276 


\begin{abstract}
Background: Adjustment disorder (AjD) is one of the most widespread mental disorders worldwide. In ICD-11, AjD is characterized by two main symptom clusters; preoccupation with the stressor and failure to adapt. The network analytic approach has been applied to most ICD-11 stress related disorders. However, no study to date explored the relations between symptoms of AjD using network analysis.

Aims: We aimed to explore AjD symptoms network and whether its structure replicates across questionnaire versions and samples.
\end{abstract}

Methods: A network analysis was conducted on AjD symptoms as assessed by the Adjustment Disorder-New Module (ADNM-8) and an ultra-brief version (ADNM-4) using data from 2,524 participants in Nigeria $(n=1006)$, Kenya $(n=1018)$, and Ghana $(\mathrm{n}=500)$.

Results: There were extensive connections between items across all samples in both ADNM versions. Results highlight that preoccupation symptoms seem to be more prominent in terms of edges strengths and had the highest centrality in all networks across samples and ADNM versions. Comparisons of network structure invariance revealed one difference between Nigeria and Ghana in both ADNM versions. Importantly, the ADNM-8 global strength was similar in all networks whereas in the ADNM-4 Kenya had a higher global strength score compared to Nigeria Conclusions: Results provide evidence of the coherence of AjD in the ICD-11 as assessed by the ADNM questionnaire. The prominence of preoccupation symptoms in AjD highlight a possible therapeutic target to alleviate distress. There is a need to further replicate the network structure of $\mathrm{AjD}$ in non-African samples. 
Keywords: ICD-11 Adjustment Disorder; Statistical Methodology; Trauma;

Individual Psychotherapy 


\section{The network structure of ICD-11 Adjustment Disorder: A cross-cultural comparison of three African countries}

\section{Adjustment disorder in ICD-11}

Adjustment disorder (AjD) has been identified as one of the most prevalent mental disorders worldwide. ${ }^{1,2}$ According to ICD- $11^{3} \mathrm{AjD}$ is a maladaptive reaction to a stressful life event, ongoing psychosocial adversities or a combination of stressful life situations that usually emerges within a month of the occurrence of a stressor and tends to resolve within six months, unless the stressor persists for a longer duration. In ICD-11, AjD is characterized by two main symptom clusters: 'preoccupations with the stressor', which includes symptoms such as recurrent and distressing thoughts or rumination about the stressor or its implications, and 'failure to adapt', which includes difficulties concentrating, sleep disturbances and an inability to recover emotionally. ${ }^{4,5}$ For a diagnosis of AjD, the symptoms must be associated with significant impairment in functioning.

\section{Operationalization of Adjustment Disorder}

The introduction of specific diagnostic criteria in ICD-11 represents a change in the conceptualization of $\mathrm{AjD}$ which previously was considered as a diagnosis if a person failed to meet criteria for another disorder. ${ }^{4}$ In parallel to the development of the $\mathrm{AjD}$ symptom criteria, a scale to assess $\mathrm{AjD}$ has been developed for validation of the newly proposed concept. Maercker, Einsle and Kollner (2007) introduced and initially validated a 29-item self-report questionnaire, the Adjustment Disorder-New Module (ADNM), which was later condensed to 20 items. $^{6}$ The ADNM-20 can be used to assess the two core symptom clusters of AjD in ICD-11 (preoccupation with the stressor and failure to adapt), as well as accessory stress-related symptoms 
(depression, anxiety, avoidance, impulsivity). Several validation studies of both ADNM versions indicated good psychometric properties (e.g., convergent and discriminant validity, factor structure, internal consistency). ${ }^{6,7}$ More recently and in line with the conceptualization of $\mathrm{AjD}$ in the ICD-11, an 8-item brief version, consisting of only the core symptoms ${ }^{8}$ and an ultra-brief measure consisting of only two items of preoccupation and two items of failure to adapt ${ }^{9}$ were produced and validated. Findings demonstrate that both the brief ADNM- 8 and the ultra-brief ADNM-4 subscales are reliable and valid instruments for the assessment of AjD. ${ }^{8,9}$

\section{Network Analytic methods}

The coherence of ICD-11 AjD has been predominantly explored using factor analytic methods, as described in the above-mentioned studies. However, factor analytic models assume a pre-determined set of factors. ${ }^{10}$ This inherent limitation of latent variable models means they are less efficient in providing the full complexity of relations among the different symptoms of $\mathrm{AjD}$. The network approach, on the other hand, conceptualizes mental disorders as systems of connected symptoms rather than reflecting an unobservable disorder. The symptoms co-occur because they reciprocally reinforce each other, not because they arise from a common underlying cause. ${ }^{10,11}$ One of the advantages of the network approach is that the interconnections of symptoms can be mathematically analyzed and visually exemplified. A network structure consists of "nodes" that represent the symptoms studied and edges that represent the relationship between nodes. Edges have thicknesses ("weights") corresponding to the strength of the association between the nodes they connect. Graph theory has been used to represent different spatial and functional characteristics that reveal information about the type of relationship between the nodes in the network. ${ }^{12}$ Visualizing AjD in this way allows insight into the complex relations 
among its symptoms and allows estimating the structure of the different measures described.

From a clinical point of view, network analytic techniques place the focus on understanding the individual symptoms of a syndrome and can identify the symptoms that are most central within the AjD network and convey high level of clinical information. ${ }^{12}$ Central symptoms are those having many strong connections to other symptoms, greater numbers of connections, and those that bridge between other symptoms. ${ }^{13}$ Identifying central symptoms of a disorder is of crucial importance to clinicians in order to guide intervention efforts. It may also identify key symptoms associated with the prognosis of patients, rather than trusting on global scores or a dichotomous diagnosis. Preliminary findings suggest that symptom centrality is related to the longitudinal course of a disorder. ${ }^{14}$ In the case of AjD, very few disorder-specific interventions have been developed to date ${ }^{15}$ and thus, obtaining information on symptom centrality may be particularly relevant for improving future treatment efforts.

The network analytic approach has been applied to most ICD-11 stress related disorders, such as complicated grief ${ }^{16}$, posttraumatic stress disorder and complex posttraumatic stress disorder. ${ }^{17}$ However, to date, AjD has not been explored using this statistical framework for its newly defined core symptoms. Considering the controversies around $\mathrm{AjD}$ and the new structure in ICD-11, it is worthwhile to explore AjD symptoms networks.

\section{The current Study}

We aimed to analyze the symptoms network of ICD-11 AjD using scales that estimate the core symptoms only, i.e., the brief ADNM-8 and the ultra-brief ADNM- 
4, in a a large dataset including three samples collected in Nigeria, Kenya and Ghana. This strategy allowed to compare the similarity of the network results in both questionnaire versions and across three different samples. We aimed to (1) assess conceptual validity by exploring which of the symptoms are strongly associated with one another and are geographically located adjacently. Support to the ICD-11 conceptualization would be reflected in stable connectivity of the network with high connections amongst preoccupation symptoms and amongst failure to adapt symptoms. Preoccupations should be associated to a lesser degree with failure to adapt symptoms (2) examine which symptoms are most central and whether they belong to the preoccupations- or the failure to adapt cluster, and (3) to explore the stability of findings between samples and ADNM versions.

\section{Methods}

\section{Participants and Procedure}

The study sample included 2,524 participants from Nigeria $(n=1,006)$, Kenya $(n=$ $1,018)$, and Ghana $(n=500)$. Each sample was drawn from a panel using stratified and random probability sampling methods to ensure a close approximation of representativeness in terms of census data on age and sex in each country (for more info regarding sample see. ${ }^{18}$ The study was approved by the institutional review board at Ariel's University [AU-MBE-2018-1029]. Each participant signed an electronic informed consent prior to participation. Inclusion criteria were citizenship of one of the following countries (Nigerian. Ghana, and Nigeria), age over 18 and having English proficiency.

\section{Measurements}


The Adjustment Disorder-New Module-8 (ADNM- $\left.8^{8}\right)$ assesses the preoccupation and failure to adapt similarly to the ICD-11. Participants first rate a list of stressors, indicating which stressors they experienced during the previous two years. Then, they rate the presence of $\mathrm{AjD}$ symptoms during the last two weeks. Four items refer to preoccupation with the stressor(s) and four items assess failure to adapt symptoms (see Table 1). Each item is scored on a 4-point Likert-type scale (1=never, $2=$ rarely, $3=$ sometimes, $4=$ often). The total score of the ADNM- 8 is the sum of responses to all items, and higher scores are indicative of greater severity of AjD. The internal reliabilities of the ADNM-8 were satisfactory for Ghana (.91), Kenya (.90) and Nigeria (.90) for the total scores as well as for the preoccupation and the failure to adapt subscales, in Ghana $(.85, .83)$, Kenya $(.85, .84)$ and Nigeria $(.86, .83)$, respectively.

The Adjustment Disorder-New Module-4 (ADNM- $\left.4^{9}\right)$ is an ultra-brief version of the ADNM-8 with a clear factor structure and good convergent and discriminant validity. It assesses AjD core symptoms (preoccupations and failure to adapt) with two items each (see Table 1). Each item is scored on a 4-point Likert-type scale (1=never, $2=$ rarely, $3=$ sometimes, $4=$ often). The score of the total ADNM- 4 is the sum of responses to all items, and higher scores are indicative of greater severity of $\mathrm{AjD}$. The internal reliability of the ADNM-4 preoccupation and failure to adapt scales were acceptable for Ghana (.82), Kenya (.83) and Nigeria (.80) samples.

\section{Statistical analysis}

\section{Regularized partial correlation networks across the three samples}

More information regarding network estimation and stability and accuracy of both edges and the centrality index techniques can be found in supplementary materials. 
Network estimation and visualization: We estimated partial pairwise correlations parameters between all nodes, through a Gaussian Graphical Model (GGM). The methodology is described in details in the supplementary materials section. We used the graphical least absolute shrinkage and selection operator (Graphical Lasso; implemented in qgraph), which visualizes sparse networks using part correlations and considered the ordinal scale of the questionnaire.

Network stability: We examined the stability of the individually estimated networks, including estimating $95 \%$ confidence intervals around the edge weights and estimating a correlation-stability coefficient for strength centrality. More information regarding the network analysis techniques can be found in supplementary materials, and in a tutorial. ${ }^{19}$

Network comparisons: To compare differences between networks, we estimated network differences between each pair of networks using the NetworkComparisonTest (NCT) package in $\mathrm{R} .{ }^{20}$ More information regarding the network comparisons techniques can be found in supplementary materials.

\section{Results}

\section{Descriptive information}

Table 1 shows the mean scores on the AjD core symptoms items across the three samples. All items differed across the three samples, although the effect size $\left(\eta^{2}\right.$ : small $=.10$, medium $=0.25$, large $=0.50)$ were generally small. The Kenyan sample had higher mean scores in all individual symptoms compared to both Ghana and Nigeria. The rates of probable AjD were high in all three countries; Ghana (23.4\%), Kenya (27.8\%), and Nigeria (17.7\%). The samples had also different number of stressors $\mathrm{F}(2,2521)=34.91 \mathrm{p}<.001 \eta^{2=} .03$ which is a medium-large effect. People from Kenya and Nigeria reported higher rates of stressors compared to Ghana, in 
particular assault, financial problems, move to a new home, unemployment, illness of loved one, and death of a loved one.

\section{Regularized partial correlation networks across the three samples}

\section{Network estimation of the ADNM-8}

Estimated networks are shown in the supplementary materials (Fig. SM1 in supplementary materials). To enhance visual comparability of edges, we estimated the average layout of the three networks and presented all networks using this layout (Fig. 1). In the ADNM-8 symptoms network, 19 of 28 possible edges $(68.8 \%)$ in the Ghana network, 21 of 28 possible edges (75.0\%) in the Kenya network, and 20 of 28 possible edges $(71.4 \%)$ in the Nigeria network were nonzero. This designates that the symptoms had extensive connections with each other in all samples. The visual inspection of the three networks exhibited many consistent edges across the samples, such as most robust connections between the preoccupation item: 'repeated thoughts' (item 1) and 'sense of burden' (item 2). Next in the hierarchy of edges strength is the association between the impairment in functioning item (item 8) and failure to adapt items (item 6 - 'difficulties doing work/tasks' and item 7 - 'sleep difficulties'). In the Nigerian sample, there was also a substantial association between the failure to adapt items 6 ('difficulties going to work/doing daily tasks'), and 7 ('sleep difficulties'), as well as a strong association between the preoccupation items 4 ('constant memories') and 5 ('thoughts often revolve'). In the Ghana sample, the preoccupation item 4 ('constant memories') was strongly associated with the failure to adapt item 3 ('difficulties concentrating').

Network stability of the ADNM-8 
To confirm the visual similarity of networks, we used Spearman correlations of edgeweights for all combinations of networks, presented in supplementary materials. Analysis shows that the accuracy of the edges was satisfactory.

The results of the confidence interval showed that edge-weights were moderately large. In addition, the results showed low accuracy of the centrality strength index (see supplementary material text, results: Network accuracy and stability and Fig. SM3-SM6).

\section{Network inference of the ADNM-8}

The standardized strength centrality estimates are presented in Fig. SM2 in supplementary material. Item 2 ('sense of burden') was the node with the highest strength centrality in all networks. However, the nodes with the smallest centrality differ between networks, though in all of them, it was from the 'failure to adapt' subscale. In the Ghana network, it was the 'difficulties going to work/doing daily tasks', in the Kenya network, it was 'sleep problems', and in the Nigeria network, it was 'constant memories'.

\section{Network comparisons of the ADNM-8}

Results from the network comparison test showed that global strength values per group were 3.56, 3.55 and 3.51 for Ghana, Kenya and Nigeria, respectively (S statistics for each pair of samples ranged 0.01 to 0.06 and $p$ value ranged .32 to .89 ). The Nigeria network structure differed from Ghana $(\mathrm{M}=.19, p=.01)$. Kenya and Ghana $(\mathrm{M}=.14, p=.28)$ as well as Kenya and Nigeria $(\mathrm{M}=.09, p=.59)$ were similar concerning structure and the level that nodes that were connected.

\section{Network estimation of the ADNM-4}

Estimated networks are shown in the supplementary materials (Fig. SM7 in supplementary materials). We estimated the average layout of the three networks and 
presented all networks using this layout (see Fig. 2). In the symptoms network of the AjD according to the ADNM-4, six of six possible edges (100\%) in the Ghana and Kenya networks, and five of six possible edges $(83.3 \%)$ in the Nigeria network were nonzero. This designates that the symptoms had highly extensive connections with each other in all samples.

The visual inspection of the three networks exhibited many similarities across the three samples, such as most robust connections between the two items of the preoccupation items and between the two items of the failure to adapt scale. The third item of difficulties in concentrating was associated with the two preoccupation items. The fourth item of difficulties with work/tasks was relatively weakly related to the failure to adapt items.

\section{Network stability of the ADNM-4}

The results of the confidence interval showed that edge-weights were moderately large. In addition, the results showed high accuracy of the centrality strength index (see supplementary material, Results: Network accuracy and stability and Fig. SM9SM12 for more details).

\section{Network inference of the ADNM-4}

Analysis shows that the accuracy of the edges is satisfactory. The standardized strength centrality estimates are presented in Fig. SM8. Item 2 ('constant memories') was the node with the highest strength centrality in all networks. In the Kenyan network, it was equally central with item 3 ('difficulties to concentrate'). In all three networks the least central item was 'difficulties in work/tasks'.

\section{Network comparison of the ADNM-4}

Global strength values per group were 1.51, 1.55 and 1.48 for Ghana, Kenya and Nigeria, respectively. Ghana did not differ from Kenya $(S=0.04 p=.40)$ and Nigeria 
$(\mathrm{S}=.03 \mathrm{p}=.53)$. The Kenyan network had a higher global strength compared to Nigeria $(\mathrm{S}=0.6 \mathrm{p}=.05)$. The Nigerian network structure differed from that of Ghana $(\mathrm{M}=.15$ $\mathrm{p}=.05)$. Kenyan and Ghana $(\mathrm{M}=.11 \mathrm{p}=.34)$ as well as Kenyan and Nigeria $(\mathrm{M}=.11$ $\mathrm{p}=.12$ ) networks were similar concerning structure and the level that nodes were connected.

\section{Discussion}

The current study investigated the symptom network structure of the ICD-11 AjD in three nationally representative samples from Nigeria, Kenya, and Ghana. To our knowledge, this was the first investigation of the $I C D-11 \mathrm{AjD}$ network structure. Results suggested extensive connections between items in all samples in the ADNM-8 and robust highly extensive associations in the ADNM-4 network. The network structure was relatively consistent across questionnaire versions and countries regarding the inter associations between nodes, and results suggest that preoccupation symptoms seem to be most central to the clinical picture of AjD. This consistency of findings provides further evidence for the conceptual validity of this newly defined condition as assessed by the ADNM questionnaire. In particular, the clinical picture arising from the findings gives support to the preoccupation symptoms as more central, while failure to adapt symptoms and functioning were intertwined.

The first aim of the study was to assess conceptual validity of AjD by exploring the individual symptoms' dynamics (i.e., evaluating which of the symptoms are strongly associated with one another). The results partially support the syndromic integrity of ICD-11 AjD as assessed by ADNM-8. Specifically, there was high connectivity between two preoccupation items - Item 1 'repeated thoughts' and Item 2 'sense of burden'. While the first symptom represents an objective observation of repeated thoughts, the second symptom represents the subjective appraisal of burden 
related to repeated thoughts. However, across all three networks, the remaining two preoccupation items (Items 4, 5) were not highly connected to the network based on partial correlations, which suggests that they do not add unique variance to the $\mathrm{AjD}$ network. Scrutinizing the phrasing of the items, it is evident that Item 1, Item 4 and Item 5 are highly similar. It is possible that these items assess the same symptom (repetitive thoughts about the stressor) rather than representing distinct symptoms of the preoccupation syndrome. In line with this explanation, both preoccupation items of the ADNM-4 (Item 2 and Item 4) were highly connected and seem to represent distinct aspects of the preoccupation syndrome, that are significant above and beyond others. This finding suggests that the ADNM-4 may be sufficient to represent preoccupations as assessed by the ADNM questionnaire and implies that some items on the 8-item version may have been redundant. This is further shown by satisfactory stability of the strength centrality in the ADNM-4 network as opposed to the poor stability of the strength centrality in the ADNM-8. Nevertheless, future research should evaluate whether they adequately cover the preoccupation cluster of AjD.

Failure to adapt items did not represent a strongly interrelated network in the ADNM-8. This finding is in line with earlier observations of acceptable but relatively low internal consistencies of the subscale, ranging from Cronbach's $\alpha=.71$ in a helpseeking sample ${ }^{8}$ to $\alpha=.80$ in a non-clinical sample exposed to burglary ${ }^{7}$. The weaker associations between different failure to adapt nodes as compared to preoccupation nodes, however, has face validity, since they cover a variety of symptoms from concentration difficulties to sleep problems. Interestingly, the analysis of the network structure revealed that two failure to adapt items (Item 6 'difficulties doing work/tasks' and Item 7 'sleep difficulties') are strongly associated with functional impairment in AjD. This finding suggests that failure to adapt symptoms as assessed by the ADNM- 
8 are more strongly associated with functional impairment compared to preoccupation symptoms. It can also imply that failure to adapt is intertwined with functional impairment, perhaps a subjective perception of it, as opposed to more actual malfunctioning.

The failure to adapt Item 3 ('concentration difficulties) was associated to an equal extent with preoccupation symptoms as with other failure to adapt symptoms. Consequently, concentration difficulties may act as a bridge symptom between the two core symptom clusters, potentially increasing the likelihood of experiencing one syndrome when experiencing the other. ${ }^{21}$ Concentration problems may be a result of both preoccupation and failure to adapt symptoms. For example, it is possible that preoccupations, such as constant, uncontrollable memories (e.g., Item 4), are a cause of concentration difficulties among patients with $\mathrm{AjD}$. At the same time, concentration difficulties may result from sleep problems (Item 7), which belongs to the failure to adapt cluster. As the network approach does not assume that the indicators of a disorder are independent, it can visualize such potentially causal relations. ${ }^{11}$ Future research is needed in order to clarify the temporal order and interdependence of AjD symptoms.

Overall, the two AjD core symptom clusters of preoccupations and failure to adapt were clearly interrelated. This finding aligns with previous factor analytical studies that lent support to a unidimensional conception of $\mathrm{AjD}$ as assessed by the ADNM. They showed high correlations between .75 to .96 between subfactors of AjD. ${ }^{6,22}$ The current study contributes this evidence by using a methodology that did not assume latent factor but rather visualizes the complex relations between symptoms. 
The second aim of the study was to examine which symptoms are most central to the AjD network and whether they belong to the preoccupations- or the failure to adapt core symptom clusters. In the ADNM-8 and ANDM-4, the node with the highest strength centrality was of the preoccupation scale. More specifically, in ADNM-8 the preoccupation Item 2 ('sense of burden') had the highest strength centrality in all three networks. This finding, however, was limited by the fact that the stability of the index was insufficient, creating some ambiguity about the centrality of this preoccupation symptom. Thus, the ADNM-4 network was explored and yet again a preoccupation item (Item 2 'constant memories') showed the most strength centrality, with high and satisfying stability. Highly central symptoms have the potential to maintain a disorder. ${ }^{23}$ The results thus highlight the relatively higher importance of preoccupation symptoms as compared to failure to adapt symptoms. This finding aligns with results from a longitudinal study over a 1-year period which showed that intrusive memories was the symptom that was most likely to be associated with a diagnosis of AjD. ${ }^{24}$

Finally, the study aimed to explore the stability of findings between samples and measures. The overall connectivity was similar across countries. However, as could be expected, there were several differences between the networks of different countries. While Nigeria and Kenya, as well as Ghana and Kenya did not differ in terms of network structure invariance, Nigeria and Ghana differed significantly in both ADNM-8 and ADNM-4. Importantly, the global score of the ADNM-8 was similar in all networks. This implies that the associations have the same magnitude of overall connectivity in all networks as well as same structure, but the edges structure is significantly different between the Nigeria and Ghana networks. This difference may be rooted in the Nigerian sample, which had stronger associations of two failure 
to adapt items (Items 6,7) than was the case in the samples from Ghana and Kenya. Moreover, among the preoccupation symptoms the Nigerian network also included stronger connections (Items 4,5 ) compared to the other samples, which indicates that both core symptom clusters were more distinctly represented in the Nigerian sample. Interestingly, studies show that Nigeria may be a particularly disadvantaged country with regard to mental health access and support. ${ }^{25}$ There was higher level of exposure to life events in Nigeria compared to Ghana and equal exposure to life events compared to Kenya ${ }^{18}$. It may be speculated that the higher prevalence of exposure may result in higher support of the AjD structure in the Nigerian network compared to Ghana.

The study has several limitations. First, it relied on self-report data rather than clinician-administered interviews, which may have biased the reports. Second, findings in community samples may not generalize to treatment-seeking or clinical samples. Third, the stability of the centrality index in the ADNM-8 networks was not high enough. However, the high stability in the ADNM-4 networks confirmed the centrality of preoccupations and made our conclusions more solid. Fourth, the crosssectional nature of the data does not allow for any inferences on causality. Fifth, the samples that were examined represented non-western cultures and it remains to be explored in replication studies whether the results generalize to western societies. However, given the rarity of studies focused on $\mathrm{AjD}$ in a non-western context, the findings also represent an important step in validating the ICD-11 AjD concept more widely.

Despite these limitations, the current findings provide an important first impression of the network structure of $\mathrm{AjD}$ and revealed patterns of association that can guide future research and practice. Despite cultural variations in samples, the 
structure of the network remained relatively consistent across all three countries. First and foremost, results provide further evidence for the validity of this newly defined condition, particularly with regard to the preoccupation syndrome. As the ICD-11 and the DSM-5 conceptualizations of AjD differ, most significantly with regard to the focus on preoccupation with the stressor in ICD-11, investigations regarding the nature of the preoccupation syndrome are crucial. The current study sheds light on the two AjD core symptom criteria of the ICD-11 and shows the complexity in the relationship between them.

One of the major benefits of defining specific symptom criteria for ICD-11 AjD is that it facilitates the development of disorder-specific interventions. ${ }^{6}$ The current study further contributes to guide future intervention development by emphasizing the central role of preoccupation with the stressor and highlighting them as particularly promising targets for intervention. In cognitive behavioral therapy, for example, an important treatment component is psychoeducation regarding functional thoughts and problems solving, on the one hand, and dysfunctional rumination, on the other hand. Furthermore, imaginal exposure-based techniques may be adequate interventions if AjD patients are oscillating between preoccupations with the stressors and attempts to avoid remembering the stressor. ${ }^{15}$ Given its central role in AjD networks across different questionnaire versions and countries, future research should aim to better understand the clinical importance of the preoccupation cluster.

\section{Funding}

An internal research grant was awarded to MBE from Ariel University (grant no. RA1700000037). The sponsor had no involvement in study design, data collection, analysis, interpretation of the results, writing of the report or the decision to submit the article for publication.

Declaration of Interest (on behalf of all authors): None. 


\section{References}

1 Evans SC, Reed GM, Roberts MC, Esparza P, Watts AD, Ritchie PLJ, et al. Psychologists' perspectives on the diagnostic classification of mental disorders: Results from the WHO-IUpsyS Global Survey. Int J Psychol 2013; 48: 177-93.

2 Reed GM, Correia JM, Esparza P, Saxena S, Maj M. The WPA-WHO global survey of psychiatrists' attitudes towards mental disorders classification. World Psychiatry 2011; 10: 118-31.

3 WHO. International classification of diseases, 11th revision. World Health Organisation, 2018.

4 Maercker A, Brewin CR, Bryant RA, Cloitre M, Ommeren M, Jones LM, et al. Diagnosis and classification of disorders specifically associated with stress: Proposals for ICD-11. World Psychiatry 2013; 12: 198-206.

5 Shevlin M, Hyland P, Ben-Ezra M, Karatzias T, Cloitre M, Vallières F, et al. Measuring ICD-11 adjustment disorder: The development and initial validation of the International Adjustment Disorder Questionnaire. Acta Psychiatr Scand 2020; 141: 265-74.

6 Glaesmer H, Romppel M, Brähler E, Hinz A, Maercker A. Adjustment disorder as proposed for ICD-11: Dimensionality and symptom differentiation. Psychiatry Res 2015; 229: 940-8.

7 Lorenz L, Bachem R, Maercker A. The Adjustment Disorder-New Module 20 as a screening instrument: Cluster analysis and cut-off values. Int J Occup Environ Med 2016; 7: 215-22.

8 Kazlauskas E, Gegieckaite G, Eimontas J, Zelviene P, Maercker A. A brief 
measure of the international classification of diseases-11 adjustment disorder: Investigation of psychometric properties in an adult help-seeking sample. Psychopathology 2018; 51: 10-15.

9 Ben-Ezra M, Mahat-Shamir M, Lorenz L, Lavenda O, Maercker A. Screening of adjustment disorder: Scale based on the ICD-11 and the Adjustment Disorder New Module. J Psychiatr Res 2018; 103: 91-6.

10 Borsboom D, Cramer AOJ. Network analysis: An integrative approach to the structure of psychopathology. Annu Rev Clin Psychol 2013; 9: 91-121.

11 Borsboom D. Psychometric perspectives on diagnostic systems. J Clin Psychol 2008; 64: 1089-108.

12 Contreras A, Nieto I, Valiente C, Espinosa R, Vazquez C. The study of psychopathology from the network analysis perspective: A systematic review. Psychother Psychosom 2019; 88: 71-83.

13 Borsboom D. A network theory of mental disorders. World Psychiatry 2017; 16: $5-13$.

14 van Borkulo CD, Boschloo L, Borsboom D, Penninx BWJH, Lourens JW, Schoevers RA. Association of symptom network structure with the course of longitudinal depression. JAMA Psychiatry 2015; 72: 1219-26.

15 Bachem R, Casey P. Adjustment disorder: A diagnose whose time has come. $J$ Affect Disord 2018; 227: 243-53.

16 Robinaugh DJ, LeBlanc NJ, Vuletich HA, McNally RJ. Network analysis of persistent complex bereavement disorder in conjugally bereaved adults. $J$ Abnorm Psychol 2014; 123: 510-22. 
17 Knefel M, Tran US, Lueger-Schuster B. The association of posttraumatic stress disorder, complex posttraumatic stress disorder, and borderline personality disorder from a network analytical perspective. J Anxiety Disord 2016; 43: 708.

18 Ben-Ezra M, Hyland P, Karatzias T, Maercker A, Hamama-Raz Y, Lavenda O, et al. Prevalence of ICD-11 disorders specifically associated with stress in Africa: A cross-country psychiatric screening of ICD-11 disorders specifically associated with stress in Kenya, Nigeria and Ghana. Eur J Psychotraumatol 2020; 11: 1720972.

19 Epskamp S, Borsboom D, Fried EI. Estimating psychological networks and their accuracy: A tutorial paper. Behav Res Methods 2018; 50: 195-212.

20 van Borkulo CD, Boschloo L, Kossakowski JJ, Tio P, Schoevers RA, Borsboom D, et al. Comparing network structures on three aspects: A permutation test. , 2017 (http://dx.doi.org/10.13140/RG.2.2.29455.38569).

21 Cramer AOJ, Waldorp LJ, Van Der Maas HLJ, Borsboom D. Comorbidity: A network perspective. Behav Brain Sci 2010; 33: 137-50.

22 Zelviene P, Kazlauskas E, Eimontas J, Maercker A. Adjustment disorder: Empirical study of a new diagnostic concept for ICD-11 in the general population in Lithuania. Eur Psychiatry 2017; 40: 20-5.

23 Hallquist MN, Wright AGC, Molenaar PCM. Problems with centrality measures in psychopathology symptom networks: Why network psychometrics cannot escape psychometric theory. Multivariate Behav Res 2019; : advance online publiction. 
24 O’Donnell ML, Alkemade N, Creamer M, McFarlane AC, Silove D, Bryant RA, et al. A longitudinal study of adjustment disorder after trauma exposure. Am J Psychiatry 2016; 173: 1231-8.

25 Odeyemi IA, Nixon J. Assessing equity in health care through the national health insurance schemes of Nigeria and Ghana: A review-based comparative analysis. Int $J$ Equity Health 2013; 12: 1-18. 
Table 1.

F statistics, Means and Standard deviations of the ADNM items of the three samples

\begin{tabular}{llllll}
\hline ADNM items & Ghana & Kenya & Nigeria & $F$ & $\eta^{2}$ \\
& & & & & \\
\hline Preoccupation & & & & & \\
\hline
\end{tabular}

Note. All $\mathrm{p}$ values are $<.001$, Means sharing a common subscript are not significantly

different at $\alpha=.01$ according to Bonferroni significant difference procedure. 

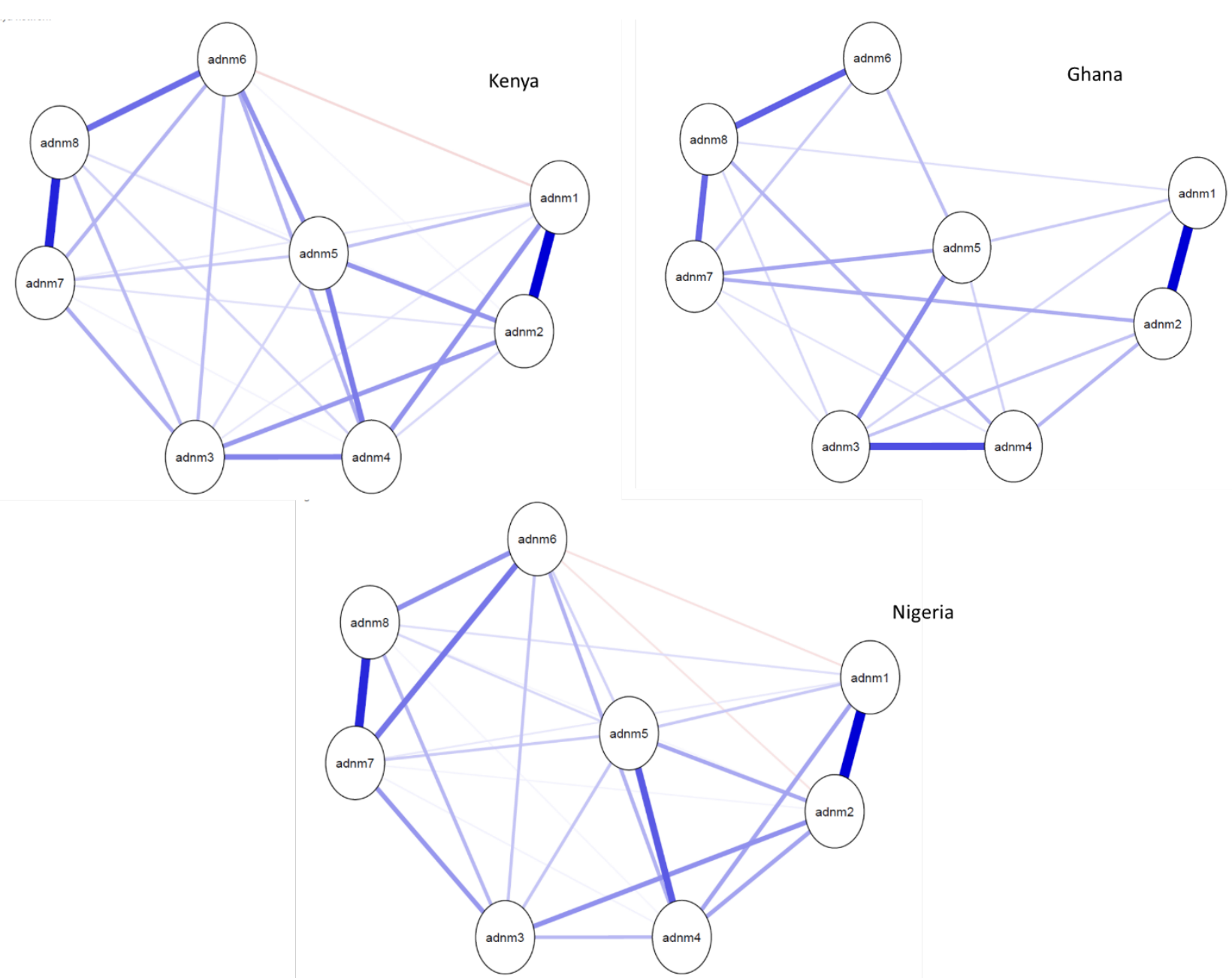

Fig 1. Networks of ADNM-8 Adjustment disorder symptoms in three African samples using average spring layout. Nodes represent ADNM-8 items, and edges Regularized partial correlations with LASSO penalty. Distances among nodes and thickness of edges relate to the size of their partial correlations. Blue edges indicate positive relations and Red edges indicate negative relationships. ADNM 1: Repeated thoughts, ADNM 2: Sense of burden; ADNM 3: Difficulties concentrating; ADNM 4: Constant memories; ADNM 5: Thoughts revolve; ADNM 6: Work/tasks difficulties; ADNM 7: Sleeping problems ADNM 8: Functional Impairment. The full items can be found in Table 1. 

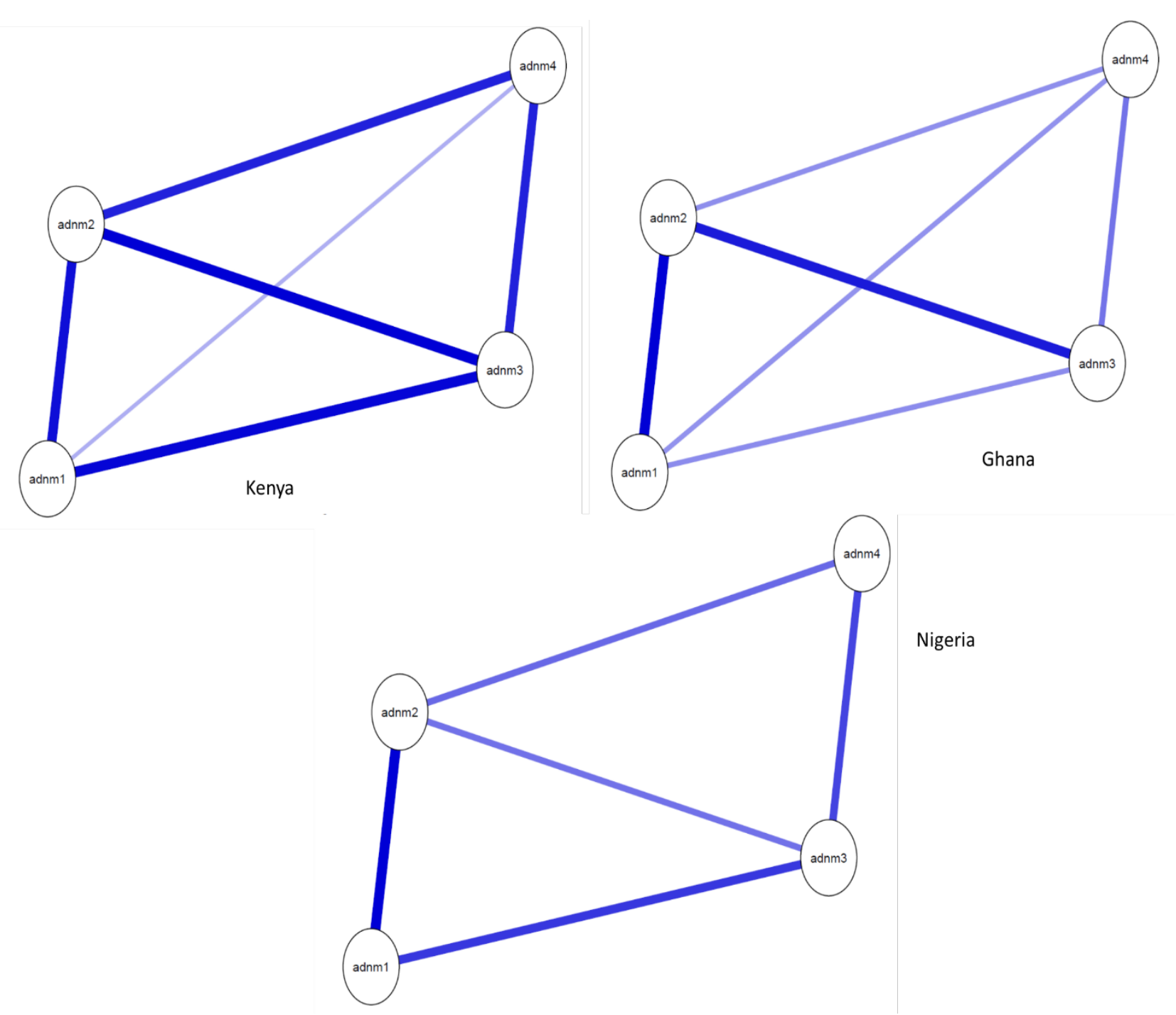

Fig 2. Networks of ADNM-4 Adjustment disorder symptoms in three African samples using average spring layout. Nodes represent ADNM-4 items, and edges Regularized partial correlations with LASSO penalty. Distances among nodes and thickness of edges relate to the size of their partial correlations. Blue edges indicate positive relations and Red edges indicate negative relationships. ADNM 1: Sense of burden; ADNM 2: Constant memories; ADNM 3: Difficulties concentrating, ADNM 4: Work/tasks difficulties. The full items can be found in Table 


\section{Authors contribution}

- Yafit Levin, PhD - Substantial contributions to the conception and design of the work, analysis, interpretation of the work, drafting and revising the work in all stages, final approval of the version to be published, and Agreement to be accountable for all aspects of the work in ensuring that questions related to the accuracy or integrity of any part of the work are appropriately investigated and resolved.

- Rahel Bachem, PhD - Substantial contributions to the conception of the work, interpretation of the work, drafting and revising the work in all stages, final approval of the version to be published, and Agreement to be accountable for all aspects of the work in ensuring that questions related to the accuracy or integrity of any part of the work are appropriately investigated and resolved.

- Thanos Karatzias, $\mathrm{PhD}$ - Substantial contributions to the design of the work, interpretation of the work, revising the work in all stages, final approval of the version to be published, and Agreement to be accountable for all aspects of the work in ensuring that questions related to the accuracy or integrity of any part of the work are appropriately investigated and resolved.

- Mark Shevlin, PhD - Substantial contributions to the design of the work, revising the work in all stages, final approval of the version to be published, and Agreement to be accountable for all aspects of the work in ensuring that questions related to the accuracy or integrity of any part of the work are appropriately investigated and resolved.

- Andreas Maercker PhD - Substantial contributions to the conception of the work, revising the work in all stages, final approval of the version to be published, and Agreement to be accountable for all aspects of the work in ensuring that questions related to the accuracy or integrity of any part of the work are appropriately investigated and resolved.

- Menachem Ben-Ezra, PhD - Substantial contributions to the conception and design of the work, revising the work in all stages, final approval of the version to be published, and Agreement to be accountable for all aspects of the work in ensuring that questions related to the accuracy or integrity of any part of the work are appropriately investigated and resolved.

\section{Data Availability Statement}

The data that support the findings of this study are available from the corresponding author, YL, upon reasonable request. 